This item was submitted to Loughborough's Research Repository by the author.

Items in Figshare are protected by copyright, with all rights reserved, unless otherwise indicated.

\title{
Two-electron elastic tunneling in low-dimensional conductors
}

PLEASE CITE THE PUBLISHED VERSION

PUBLISHER

(C) American Physical Society

LICENCE

CC BY-NC-ND 4.0

REPOSITORY RECORD

Alexandrov, A.S., A.M. Bratkovsky, and P.E. Kornilovitch. 2019. "Two-electron Elastic Tunneling in Lowdimensional Conductors". figshare. https://hdl.handle.net/2134/1321. 


\title{
Two-electron elastic tunneling in low-dimensional conductors
}

\author{
A. S. Alexandrov, ${ }^{1,2}$ A. M. Bratkovsky, ${ }^{1}$ and P. E. Kornilovitch ${ }^{1}$ \\ ${ }^{1}$ Hewlett-Packard Laboratories, 1501 Page Mill Road, MS 1L-12, Palo Alto, California 94304 \\ ${ }^{2}$ Department of Physics, Loughborough University, Loughborough LE11 3TU, United Kingdom
}

(Received 8 January 2002; published 8 April 2002)

\begin{abstract}
We solve the Lippmann-Schwinger equation describing one-dimensional elastic scattering of preformed pairs (e.g., bipolarons) off a short-range scattering center, and find the two-particle transmission through a thin potential barrier. While the pair transmission is smaller than the single-electron transmission in the strongcoupling limit, it is remarkably larger in the weak-coupling limit. We also calculate current-voltage characteristics of a molecule-barrier-molecule junction. They show unusual temperature and voltage behaviors which are experimentally verifiable at low temperatures in bulk and nanoscale molecular conductors.
\end{abstract}

DOI: 10.1103/PhysRevB.65.155209

PACS number(s): 71.38.Mx, 72.10.Fk, 73.63.Nm, 85.65.th

Molecular-scale electronics is currently a very active area of research. ${ }^{1}$ It is envisaged that linear conjugated molecules would be used as the "transmission lines" in molecular circuitry $^{2,3}$ in addition to active molecular elements discussed in the literature." When a so-called "molecular wire" is short, the dominant mechanism of transport is most likely a resonant tunneling through electronic molecular states (see Refs. 5 and 6, and references therein). With an increasing size of the wires one has to take into account the strong interaction between carriers and vibronic excitations of the molecule. For longer wires containing more than about 40 atomic sites, the tunneling time is comparable to or larger than the characteristic phonon times, so that the polaron (and/or bipolaron) can be formed inside the molecular wire. ${ }^{7}$ There is also a wide range of molecular bulk conductors with (bi)polaronic carriers. The formation of polarons (and charged solitons) in polyacetylene (PA) was discussed a long time ago theoretically in Ref. 8, and the formation of bipolarons (bound states of two polarons) was discussed in Ref. 9. Polarons in PA were detected optically in Ref. 10, and studied since then in great detail. There is an exceeding amount of evidence of polaron and bipolaron formation in conjugated polymers such as polyphenylene, polypyrrole, polythiophene, polyphenylene sulfide, ${ }^{11}$ Cs-doped biphenyl, ${ }^{12} n$-doped bithiophene, ${ }^{13}$ polyphenylenevinylene based light-emitting diodes, ${ }^{14}$ and other molecular systems. In many cases the doped polymers have bipolaronlike charge states, to yield, in particular, enhanced nonlinear optical properties. $^{15}$

Many experimental data provide evidence of hopping transport of (bi)polaronic carriers. However, at sufficiently low temperatures there should be a crossover to the band motion of polarons, as suggested a long ago, ${ }^{16,17}$ and bipolarons. ${ }^{18,19}$ Indeed, due to recent extraordinary improvements in the preparation of organic molecular films, it has become possible to measure their conductivity in a wide interval of temperatures, and to observe the crossover in twodimensional films of organic conjugated molecules. ${ }^{20}$ In onedimensional wires the band motion is expected to be strongly hindered by imperfections, and those imperfections are likely to be intentionally introduced in the system as functionalizing units. ${ }^{21}$ Moreover, polarons in extended molecular wires or units are expected to be bound into real-space bipolarons with lowering temperature. It is known in the context of oxide semiconductors that the bipolaron formation may strongly affect transport properties. ${ }^{18,22}$

In this Brief Report we study the elastic scattering of carriers bound into real-space pairs in one-dimensional organic and other conductors. We present an exact analytical solution in the limit of slow pairs. We also find an unusual temperature and voltage dependence of the tunneling conductance which may be experimentally verified at low temperatures in organic bulk conductors and long nanowires.

In mathematical terms, the scattering of pairs is a threebody problem with the scattering potential ascribed to the third particle with an infinite mass. Let $\hat{U}\left(x_{1}-x_{2}\right)$ be an attractive potential between two moving particles, and $\hat{V}\left(x_{1}, x_{2}\right)$ a repulsive external potential representing the barrier. The starting point is the Lippmann-Schwinger equation $^{23,24}$ for the two-particle wave function $\Psi\left(k_{1}, k_{2}\right)$ in a momentum representation, which explicitly takes into account a boundary condition of the three-body scattering problem. It can be written as

$$
\Psi=-i \gamma \hat{G}(E+i \gamma) \Phi
$$

where $\hat{G}(E+i \gamma)$ is the exact two-particle Green's function $(\mathrm{GF})$ in the external potential, $\Phi\left(k_{1}, k_{2}\right)$ is the wave function of a free $(\hat{V}=0)$ real-space pair in the momentum representation, and $\Phi\left(k_{1}, k_{2}\right)=2 \pi \delta(q-Q) \phi(k)$. Here $q=k_{1}+k_{2}$ is the center-of-mass momentum, $k=\left(k_{1}-k_{2}\right) / 2$ is the relative momentum, $E=-\epsilon+Q^{2} / 4<0$ is the pair total energy in the absence of the external potential, and $\epsilon$ is its binding energy. The wave function $\phi(k)$ describes the internal structure of the pair. Hereafter we choose $\hbar=k_{B}=m_{1}=m_{2}=1$, and $\gamma=$ +0 , and define $\hat{G}(E+i \gamma) \Phi$ as

$$
\hat{G} \Phi \equiv \iint \frac{d k_{1}^{\prime} d k_{2}^{\prime}}{(2 \pi)^{2}} G\left(k_{1}, k_{2} \mid k_{1}^{\prime}, k_{2}^{\prime} ; E\right) \Phi\left(k_{1}^{\prime}, k_{2}^{\prime}\right)
$$

for any $\hat{G}$ and $\Phi$. Using the two identities $\hat{G}=\hat{G}_{3}-\hat{G}_{3} \hat{U} \hat{G}$ and $\hat{G}=\hat{G}_{12}-\hat{G}_{12} \hat{V} \hat{G}$ and the Lippmann-Schwinger equation, one readily derives the equation for the Fourier component $T\left(k_{1}, k_{2}\right)$ of the product $\hat{U} \Psi{ }^{24}$ 


$$
T\left(k_{1}, k_{2}\right)=\left(E-k^{2}-q^{2} / 4\right) \Phi-\hat{T}_{12} \Delta \hat{G}_{3} T,
$$

where $\Delta \hat{G}_{3}=\hat{G}_{3}-\hat{G}_{0}, \hat{G}_{0}$ is the two-particle GF in the absence of any interaction $(\hat{U}=\hat{V}=0), \hat{G}_{3}$ is the GF of noninteracting particles in the external field $\hat{V}$ (for $\hat{U}=0$ ), and $\hat{G}_{12}$ is the GF of two interacting particles with no external field $(\hat{V}=0)$. Here the scattering operator $\hat{T}_{12}$, defined by the relation $\hat{T}_{12} \hat{G}_{0} \equiv \hat{U} \hat{G}_{12}$, is expressed via the particle-particle scattering $t$ matrix as

$$
T_{12}\left(k_{1}, k_{2} \mid k_{1}^{\prime}, k_{2}^{\prime} ; E\right)=2 \pi t\left(k, k^{\prime} ; E-q^{2} / 4\right) \delta\left(q-q^{\prime}\right) .
$$

The $t$ matrix satisfies the equation

$$
t\left(k, k^{\prime} ; E\right)=u\left(k-k^{\prime}\right)-\int \frac{d p}{2 \pi} \frac{u(k-p) t\left(p, k^{\prime} ; E\right)}{p^{2}-E-i \gamma},
$$

where $u(p)$ is the Fourier component of the attractive potential $\hat{U}\left(x_{1}-x_{2}\right)$.

In many (in)organic semiconductors, the long-range Coulomb repulsion is usually significantly reduced by the strong Fröhlich interaction with optical phonons, ${ }^{25}$ so that a net (attractive) potential between carriers is a short-range one, $\hat{U}\left(x_{1}-x_{2}\right)=-\alpha \delta\left(x_{1}-x_{2}\right), \alpha>0$. Then Eq. (5) is readily solved, resulting in the momenta-independent $t$ matrix

$$
t\left(k, k^{\prime}, E\right)=-\frac{\alpha \sqrt{-E}}{\sqrt{-E}-\alpha / 2},
$$

which is valid for all energies provided that the square root is understood as its principal value. The binding energy is $\epsilon$ $=\alpha^{2} / 4$, and the normalized ground-state wave function is $\phi(k)=2^{-1 / 2} \alpha^{3 / 2} /\left(k^{2}+\epsilon\right)$. It is known that for a short-range inter particle interaction $T\left(k_{1}, k_{2}\right)$, Eq. (3) is proportional to the Fourier component of the center-of-mass wave function $\Omega(q), T\left(k_{1}, k_{2}\right)=-2^{-1 / 2} \alpha^{3 / 2} \Omega(q)$. Then the problem of elastic pair scattering is reduced to a single integral equation for the center-of-mass scattering amplitude $Y(q)$. Substituting $\Phi$ and $\hat{T}_{12}$ in Eq. (3), one obtains

$$
\Omega(q)=2 \pi \delta(q-Q)-\frac{\Upsilon(q)}{q^{2} / 4-Q^{2} / 4-i \gamma},
$$

where $\Upsilon(q)$ satisfies

$$
\Upsilon(q)=W(q, Q)-\int \frac{d q^{\prime}}{2 \pi} \frac{W\left(q, q^{\prime}\right) \Upsilon\left(q^{\prime}\right)}{q^{\prime}+4-Q^{2} / 4-i \gamma} .
$$

The effective center-of-mass scattering potential $W\left(q, q^{\prime}\right)$ is determined using the GF of two noninteracting particles in the external potential $(\hat{U}=0$ but $\hat{V} \neq 0$ ) as

$W\left(q, q^{\prime}\right)=\alpha \chi(q) \iint \frac{d k_{2} d k_{2}^{\prime}}{(2 \pi)^{2}} \Delta G_{3}\left(q-k_{2}, k_{2} \mid q^{\prime}-k_{2}^{\prime}, k_{2}^{\prime} ; E\right)$, where $\chi(q)=E-q^{2} / 4+(\alpha / 4)\left(q^{2}-4 E\right)^{1 / 2}$. In the following we restrict our consideration to the scattering of slow pairs with $Q^{2} \ll 4 \epsilon$. This condition allows us to replace $W\left(q, q^{\prime}\right)$ with $W(0,0) \equiv W$ in all equations, because the characteristic momenta $q, q^{\prime} \simeq Q$ are much smaller than $\sqrt{-E}$. Then the solution of Eq. (8) is given by $Y(q)=W Q /(Q+2 i W)$, so that the pair transmission probability is

$$
\mathcal{T}_{2}(Q)=1-\left|\frac{2 \Upsilon(-Q)}{Q}\right|^{2}=\frac{Q^{2}}{Q^{2}+4 W^{2}} .
$$

In general, $G_{3}, W$, and $\mathcal{T}_{2}$ can be found only numerically. However, in many applications the scattering potential is also a short-range one, $\hat{V}\left(x_{1}, x_{2}\right)=\beta\left[\delta\left(x_{1}\right)+\delta\left(x_{2}\right)\right]$, so that the full Hamiltonian takes the form

$$
H=-\frac{1}{2} \frac{\partial^{2}}{\partial x_{1}^{2}}-\frac{1}{2} \frac{\partial^{2}}{\partial x_{2}^{2}}-\alpha \delta\left(x_{1}-x_{2}\right)+\beta\left[\delta\left(x_{1}\right)+\delta\left(x_{2}\right)\right] .
$$

The approximation of the interaction between the particles by the $\delta$ function is justified if the size of the pair is large compared with the range of the attractive potential. This is valid for bipolarons, if their size is larger than the lattice constant, and for the deuteron in nuclear physics. Analogously, approximating the barrier with the $\delta$ function is appropriate if the range of the scattering potential is small compared with the size of the pair. This three-body problem (with the infinite mass of a third particle) was considered before in Refs. 26 and 27 but no general analytical solution was found. Here we present an analytical solution in the limit of slow pairs. Consider the equation for the two-particle Green's function $G_{3}$,

$$
\begin{aligned}
& \left(k_{1}^{2} / 2+k_{2}^{2} / 2-E\right) G_{3}\left(k_{1}, k_{2} \mid k_{1}^{\prime}, k_{2}^{\prime} ; E\right) \\
& \quad+\beta \int \frac{d p_{1}}{2 \pi} G_{3}\left(p_{1}, k_{2} \mid k_{1}^{\prime}, k_{2}^{\prime} ; E\right)+\beta \int \frac{d p_{2}}{2 \pi} G_{3} \\
& \quad \times\left(k_{1}, p_{2} \mid k_{1}^{\prime}, k_{2}^{\prime} ; E\right)=(2 \pi)^{2} \delta\left(k_{1}-k_{1}^{\prime}\right) \delta\left(k_{2}-k_{2}^{\prime}\right),
\end{aligned}
$$

which has a formal solution

$$
\begin{aligned}
G_{3}\left(k_{1}, k_{2} \mid k_{1}^{\prime}, k_{2}^{\prime} ; E\right)= & G_{0}\left(k_{1}, k_{2} \mid k_{1}^{\prime}, k_{2}^{\prime} ; E\right) \\
& -\frac{D\left(k_{2} \mid k_{1}^{\prime}, k_{2}^{\prime} ; E\right)+D\left(k_{1} \mid k_{2}^{\prime}, k_{1}^{\prime} ; E\right)}{k_{1}^{2} / 2+k_{2}^{2} / 2-E} .
\end{aligned}
$$

Here

$$
\begin{aligned}
G_{0} & \left(k_{1}, k_{2} \mid k_{1}^{\prime}, k_{2}^{\prime} ; E\right) \\
\quad & =(2 \pi)^{2} \delta\left(k_{1}-k_{1}^{\prime}\right) \delta\left(k_{2}-k_{2}^{\prime}\right)\left(k_{1}^{2} / 2+k_{2}^{2} / 2-E\right)^{-1},
\end{aligned}
$$

and

$$
D\left(k_{1} \mid k_{2}^{\prime}, k_{1}^{\prime} ; E\right) \equiv(2 \pi)^{-1} \beta \int d k_{2} G_{3}\left(k_{1}, k_{2} \mid k_{1}^{\prime}, k_{2}^{\prime} ; E\right)
$$

satisfies the integral equation 


$$
\begin{aligned}
& D\left(k_{1} \mid k_{2}^{\prime}, k_{1}^{\prime} ; E\right)\left[1+\frac{\beta}{\left(k_{1}^{2}-2 E\right)^{1 / 2}}\right] \\
& =\frac{2 \pi \beta \delta\left(k_{1}-k_{1}^{\prime}\right)}{k_{1}^{2} / 2+k_{2}^{\prime 2} / 2-E}-\beta \int \frac{d k_{2}}{2 \pi} \frac{D\left(k_{2} \mid k_{1}^{\prime}, k_{2}^{\prime} ; E\right)}{k_{1}^{2} / 2+k_{2}^{2} / 2-E} .
\end{aligned}
$$

We are interested in $W=\alpha^{3}(2 \pi)^{-2} \iint d k d p D(k \mid-p, p ; E)$ $\times\left(k^{2}-E\right)^{-1}$. Integrating Eq. (14) with respect to $k_{2}^{\prime}=-k_{1}^{\prime}$ $\equiv-p$, for $B(k ; E) \equiv(2 \pi)^{-1} \int d p D(k \mid-p, p ; E)$ one obtains the following equation:

$$
\begin{aligned}
& B(k ; E)\left[1+\frac{\beta}{\left(k^{2}-2 E\right)^{1 / 2}}\right]+\beta \int \frac{d k^{\prime}}{2 \pi} \frac{B\left(k^{\prime} ; E\right)}{k^{2} / 2+k^{\prime 2} / 2-E} \\
& \quad=\frac{\beta}{k^{2}-E} .
\end{aligned}
$$

It has the solution

$$
B(k ; E)=\frac{\beta}{\left(k^{2}-E\right)\left[1+\beta(-E)^{-1 / 2}\right]},
$$

which is verified by direct substitution into Eq. (15). Finally, we obtain

$$
W=\alpha^{3} \int \frac{d k}{2 \pi} \frac{B(k ; E)}{k^{2}-E}=\frac{2 \alpha \beta}{\alpha+2 \beta} .
$$

This result, together with Eq. (10), solves the problem of the elastic scattering of slow bound pairs for any strength of the short-range attractive, potential $\alpha$ and scattering potential $\beta$. The "slow-particle" approximation used here $\left(q, q^{\prime} \simeq Q\right.$ $\ll \alpha$ ) restricts only the temperature and/or the voltage range. It is instructive to compare the pair transmission $\mathcal{T}_{2}(Q)$ [Eq. (10)], with the single-electron transmission $\mathcal{T}_{1}(p)=p^{2} /\left(p^{2}\right.$ $+\beta^{2}$ ) for equal kinetic energies $p^{2} / 2=Q^{2} / 4 \equiv K$. If the binding potential is strong compared with the scattering potential $(\alpha \gg 2 \beta)$, the pair transmission is just the single-particle transmission of a particle with a double mass and doublebarrier strength, $\mathcal{T}_{2}(Q)=Q^{2} /\left(Q^{2}+16 \beta^{2}\right)$, in accordance with a naive expectation. In the general case the ratio is

$$
\frac{\mathcal{T}_{2}(Q)}{\mathcal{T}_{1}(p)}=\frac{K+\beta^{2} / 2}{K+4 \beta^{2}(1+2 \beta / \alpha)^{-2}} .
$$

When the binding potential is weaker than the scattering potential $(\alpha \ll \beta)$, the ratio is

$$
\frac{\mathcal{T}_{2}(Q)}{\mathcal{T}_{1}(p)}=\frac{1}{2}\left(\frac{\beta}{\alpha}\right)^{2} \gg 1
$$

Quite remarkably, a weak attraction between carriers helps the first transmitted particle to "pull" its partner through a strong potential barrier.

Another important difference between pair and singleelectron tunneling occurs due to their different statistics. While electrons are fermions, preformed pairs are bosons, so that their center-of-mass motion obeys Bose-Einstein statistics. Hence tunneling conductance should be temperature dependent even at low temperatures $T$, as already established in bipolaron tunneling to a normal metal with a decay of the bound state. ${ }^{28}$ Here we calculate the current-voltage characteristics of a molecule-barrier-molecule (MBM) junction, i.e., the current through a thin potential barrier between two bulk molecular conductors with preformed pairs. For simplicity, we restrict our calculations to the strong-coupling regime, $\alpha \gg \beta, T^{1 / 2}$. In this regime, single carriers are frozen out, and the transmission is due to the pairs alone, which are scattered off a double-strength barrier $W \approx 2 \beta$ [Eq. (17)], similar to single particles with a double-carrier mass. Then, in the presence of a voltage drop at the junction, $2 \mathrm{eV}$ (for a pair), the conductance can be readily found by matching the center-of-mass wave function and its derivatives, $\Omega_{l}$, on the left side, and $\Omega_{r}$, on the right side, of the $\delta$-function barrier. In the coordinate representation one has $\Omega_{l}(X<0)=e^{i Q X}$ $+R e^{-i Q X}$, and $\Omega_{r}(X>0)=C e^{i P_{+} X}$ with $1+R=C, C P_{+}$ $-(1-R) Q=8 i \beta(1+R)$, and $P_{+}=\left(Q^{2}+8 e V\right)^{1 / 2}$. The transmission is given by

$$
\mathcal{T}_{2}\left(Q, P_{+}\right) \equiv 1-|R|^{2}=\frac{4 Q P_{+}}{\left(Q+P_{+}\right)^{2}+64 \beta^{2}}
$$

for real $P_{+}$, and is zero otherwise. Multiplying the transmission by $e Q$ and integrating with the Bose-Einstein distribution function $f(Q)=\left[\exp \left(Q^{2} / 4 T-\mu / T\right)-1\right]^{-1}$ yields the current as

$$
I(V)=e \int_{0}^{\infty} \frac{d Q}{2 \pi} Q f(Q)\left[\mathcal{T}_{2}\left(Q, P_{+}\right)-\mathcal{T}_{2}\left(Q, P_{-}\right)\right],
$$

where $P_{-}=\left(Q^{2}-8 e V\right)^{1 / 2}$, and $\mu$ is the chemical potential determined by the number of pairs $n$ using $\int_{-\infty}^{\infty}(d Q / 2 \pi) f(Q)=n$.

It is easy to calculate the integrals in the linear voltage classical limit of $2 e V, T_{F} \ll T$, by expanding the transmission in powers of $e V$ and replacing the Bose-Einstein distribution with the Boltzmann one: $f(Q) \approx\left(2 T_{F} / \pi T\right)^{1 / 2} \exp \left(-Q^{2} / 4 T\right)$ ( $T_{F} \equiv \pi^{2} n^{2} / 2$ is the Fermi temperature of single carriers). We believe that this limit is also applied to sufficiently long molecular wires, where bipolarons have sufficient time to form. The result for the conductance, $\sigma \equiv(d I / d V)_{V=0}$, is

$$
\sigma=\frac{2 e^{2}}{\pi} \sqrt{\frac{2 T_{F}}{\pi T}}\left[1+\frac{4 \beta^{2}}{T} e^{4 \beta^{2} / T} \operatorname{Ei}\left(-4 \beta^{2} / T\right)\right],
$$

where $\operatorname{Ei}(x)$ is the exponential integral function. The conductance behaves as $\sigma=\left(e^{2} / \pi \beta^{2}\right) \sqrt{T_{F} T / 2 \pi}$ at $T \ll 4 \beta^{2}$, and as $\sigma=\left(2 e^{2} / \pi\right) \sqrt{2 T_{F} / \pi T}$ at $T \gg 4 \beta^{2}$. In the latter case it has a universal magnitude independent of the barrier strength. Apart from numerical coefficients, the conductance of tightly bound pairs is, of course, the same as the conductance of single electrons in the classical limit. This is not the case, however, in a degenerate system, when $T \leqslant T_{F}$. A numerical integration of Eq. (21) at a fixed density $n$ reveals a temperature dependence in this limit, (Fig. 1), in comparison with the 


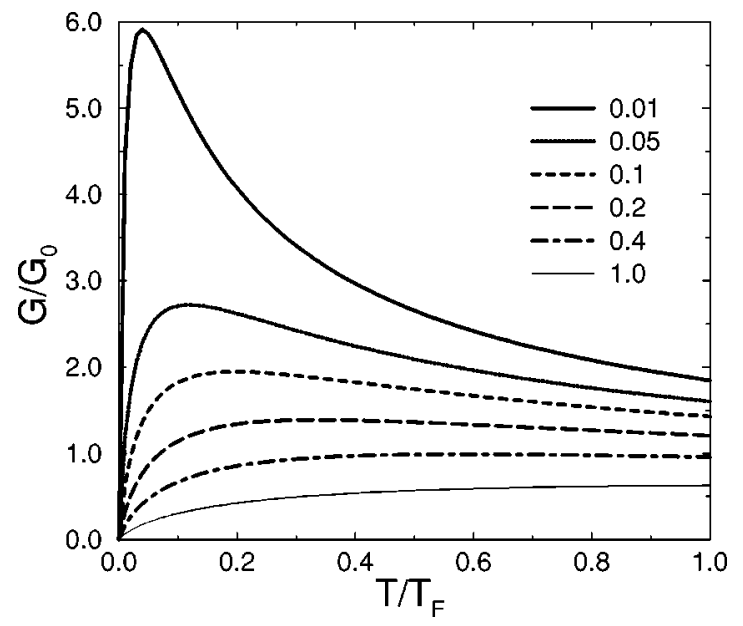

FIG. 1. Zero-voltage conductance of the MBM as a function of temperature (in units of $T_{F}$ ) for different relative strengths of the barrier $4 \beta^{2} / T_{F} \cdot G_{0}=\left(2 e^{2}\right) / h$.

temperature, independent conductance of fermionic noninteracting carriers at low temperatures. This remarkable difference is entirely due to the bosonic nature of pairs that is an effect of quantum statistics. The conductance is proportional to the mean velocity of carriers which in the case of bosons grows as $\sqrt{T}$ (while it is temperature independent for fermions). This explains the low-temperature behavior of the conductance. Interpair correlations may reduce the difference in 1D wires. However, higher-dimension corrections readily restore it. There is also a breakdown of Ohm's law when $2 e V \geqslant T$, as shown in Fig. 2 at low temperatures, again in contrast with the Fermi statistics, where a nonlinearity appears only at $e V \geqslant T_{F} \gg T$. We suggest that the most appropriate materials for experimental observation of the unusual current-voltage characteristics (Figs. 1 and 2) are doped molecular semiconductors such as Cs-doped biphenyl, ${ }^{12}$ where bipolarons were explicitly detected by photoelectron and electron-energy-loss spectroscopies, and single crystals of

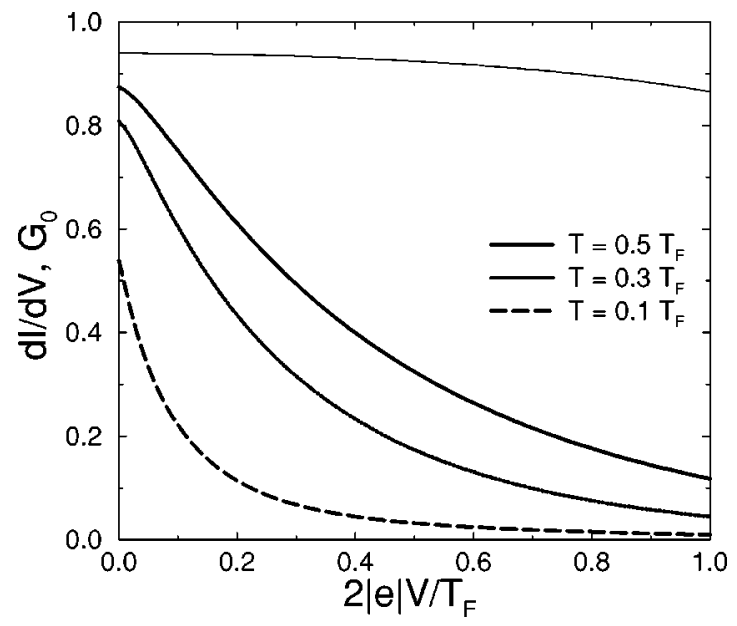

FIG. 2. Differential conductance of the MBM as a function of voltage for different temperatures and $4 \beta^{2} / T_{F}=0.5$. The thin solid lines is the conductance of fermions at $T=0.1 T_{F}$.

pentacene, tetracene, rubrene, quaterthiophene $(\alpha-4 \mathrm{~T})$, and sexithiophene $(\alpha-8 \mathrm{~T})$, where coherent polaron tunneling was recently observed below room temperature. ${ }^{20}$

In conclusion, we have solved the Lippmann-Schwinger equation in the effective-mass approximation for preformed pairs in 1D conductors (molecular wires), which is valid for (bi)polarons, if their size is larger than the lattice constant. We have calculated the amplitude of elastic scattering of slow bipolarons and the conductance of a MBM junction with preformed real-space pairs. While the pair transmission is smaller than the single-electron transmission in the strongcoupling regime, it is unexpectedly larger in the weakcoupling regime. The current-voltage characteristics of the MBM junction show unusual temperature and nonlinear voltage behavior; see Figs. 1 and 2 .

We acknowledge interesting discussions with J. P. Keating, V. V. Osipov, and R. S. Williams.
${ }^{1}$ Molecular Electronics: Science and Technology, edited by A. Aviram and M. Ratner (Ann. New York Acad. Sci., New York, 1998), Vol. 852.

${ }^{2}$ J.-M. Lehn, Angew. Chem. Int. Ed. Engl. 29, 1304 (1990).

${ }^{3}$ J.M. Tour, Acc. Chem. Res. 33, 791 (2000); J.M. Tour, L. Jones, D.L. Pearson, J.J.S. Lamba, T.P. Burgin, G.M. Whitesides, D.L. Allara, A.N. Parikh, and S.V. Atre, J. Am. Chem. Soc. 117, 9529 (1995).

${ }^{4}$ C.P. Collier, E.W. Wong, M. Belohradsky, F.M. Raymo, J.F. Stoddart, P.J. Kuekes, R.S. Williams, and J.R. Heath, Science $\mathbf{2 8 5}$, 391 (1999); J. Chen, M.A. Reed, A.M. Rawlett, and J.M. Tour, ibid. 286, 1550 (1999); D.I. Gittins, D. Bethell, D.J. Schiffrin, and R.J. Nichols, Nature (London) 408, 677 (2000).

${ }^{5}$ A. Aviram and M.A. Ratner, Chem. Phys. Lett. 29, 257 (1974); S. Datta, W.D. Tian, S.H. Hong, R. Reifenberger, J.I. Henderson, and C.P. Kubiak, Phys. Rev. Lett. 79, 2530 (1997).

${ }^{6}$ P.E. Kornilovitch and A.M. Bratkovsky, Phys. Rev. B 64, 195413
(2001).

${ }^{7}$ N. Ness, S.A. Shevlin, and A.J. Fisher, Phys. Rev. B 63, 125422 (2001).

${ }^{8}$ W.P. Su and J.R. Schrieffer, Proc. Natl. Acad. Sci. U.S.A. 77, 5626 (1980).

${ }^{9}$ S.A. Brazovskii and N.N. Kirova, Pis'ma Zh. Éksp. Teor. Fiz. 33, 6 (1981) [JETP Lett. 33, 4 (1981)].

${ }^{10}$ A. Feldblum, J.H. Kaufman, S. Etemad, A.J. Heeger, T.-C. Chung and A.G. MacDiarmid, Phys. Rev. B 26, 815 (1982).

${ }^{11}$ R.R. Chance, J.L. Bredas, and R. Silbey, Phys. Rev. B 29, 4491 (1984).

${ }^{12}$ M.G. Ramsey, D. Steinmüller, and F.P. Netzer, Phys. Rev. B 42, 5902 (1990).

${ }^{13}$ D. Steinmuller, M.G. Ramsey, and F.P. Netzer, Phys. Rev. B 47, 13323 (1993).

${ }^{14}$ L.S. Swanson, J. Shinar, A.R. Brown, D.D.C. Bradley, R.H. Friend, P.L. Burn, A. Kraft, and A.B. Holmes, Synth. Met. 55, 
241 (1993).

${ }^{15}$ C.W. Spangler, M.Q. He, E.G. Nickel, J. Laquindanum, L.R. Dalton, N.S. Tang, and R. Hellwarth, Mol. Cryst. Liq. Cryst. 240, 17 (1994).

${ }^{16}$ T. Holstein, Ann. Phys. (N.Y.) 8, 325 (1959); 8, 343 (1959).

${ }^{17}$ I.G. Lang and Yu.A. Firsov, Zh. Éksp. Teor. Fiz. 43, 1843, (1962) [ Sov. Phys. JETP 16, 1301 (1963)].

${ }^{18}$ A.S. Alexandrov and N.F. Mott, Polarons and Bipolarons (World Scientific, Singapore, 1995).

${ }^{19}$ J. Bonca, T. Katrasnik, and S.A. Trugman, Phys. Rev. Lett. 84, 3153 (2000).

${ }^{20}$ J.H. Schön, C. Kloc, and B. Batlogg, Phys. Rev. Lett. 86, 3843 (2001).
${ }^{21}$ J. Chen, W. Wang, M.A. Reed, A.M. Rawlett, D.W. Price, and J.M. Tour, Appl. Phys. Lett. 77, 1224 (2000).

${ }^{22}$ A.S. Alexandrov and A.M. Bratkovsky, Phys. Rev. Lett. 82, 141 (1999).

${ }^{23}$ B. Lippmann and J. Schwinger, Phys. Rev. 79, 469 (1950).

${ }^{24}$ A.I. Baz', Ya.B. Zeldovich, and A.M. Perelomov, Scattering, Reactions, and Decays in Nonrelativistic Quantum Mechanics (Nauka, Moscow, 1971).

${ }^{25}$ A.S. Alexandrov, Phys. Rev. B 61, 12315 (2000).

${ }^{26}$ S.L. Schwebel, Phys. Rev. 103, 814 (1956).

${ }^{27}$ J.R. Jasperse and M.H. Friedman, Phys. Rev. B 159, 69 (1967).

${ }^{28}$ A.S. Alexandrov, M.P. Kazeko, and S.G. Rubin, Zh. Éksp. Teor. Fiz. 98, 1656 (1990) [Sov. Phys. JETP 71, 1656 (1990)]. 